\title{
Platelet Transfusion Induces Alloimmunization to D and Non-D Rhesus Antigens
}

\author{
Johanna Reckhaus $^{a}$ Markus Jutzi ${ }^{b}$ Stefano Fontana ${ }^{b}$ Vera Ulrike Bacher ${ }^{a}$ Marco Vogt $^{a}$ \\ Michael Daslakis ${ }^{a}$ Behrouz Mansouri Taleghani ${ }^{a}$ \\ a University Department of Hematology, Inselspital, University of Bern, Bern, Switzerland; \\ bInterregional Blood Transfusion Swiss Red Cross, Bern, Switzerland
}

\section{Keywords}

Platelet transfusion - Rhesus alloimmunization . Hemovigilance

\section{Summary}

Background: Platelet concentrates (PC) contain residual contaminating red blood cells (RBC), being higher in pooled buffy coat PC (BC-PC) than in apheresis units (AP$P C)$. Data about $P C$-induced alloimmunization against non-D Rhesus (Rh) antigens are limited. Methods: For all newly detected RhD and non-D alloantibodies between August 2015 and September /2017 we prospectively evaluated if they were triggered through PC by analyzing for incompatible RBC and/or PC transfusions. Results: We found 5,799 positive results in 89,190 antibody screening tests. We identified 13 newly detectable Rh antibodies through incompatible PCs in 11 patients: $6 x$ anti-D, 4x anti-E, $2 \times$ anti-C, $1 \times$ anti-f. They received a total of 156 PC (83 BC-PC; 73 AP-PC): 5 patients received incompatible $\mathrm{BC}-\mathrm{PC}$ only, 1 patient received incompatible AP-PC only, 5 patients received incompatible BC-PC and AP-PC. Quality control showed a mean (range) of $0.304(0.152-1.662)$ and $0.014(0.003-0.080) \times 10^{9} \mathrm{RBC} / \mathrm{l}$ for BC-PC and AP-PC, respectively. Ten of the 11 patients received $\mathrm{RBC}$ transfusions, all of them being antigen-negative for the alloantibodies identified. Conclusions: PC transfusions may not only induce RhD alloimmunization, but also immunization against further $R$ antigens such as $C, E$, and $f$. The risk seems higher for BC-PC than for AP-PC. The results may have impact on future recommendations of $P C$ transfusion with respect to $\mathrm{Rh}$ compatibility and upper limits of RBC contamination.

(C) 2018 S. Karger GmbH, Freiburg

\section{Introduction}

Platelet concentrates (PC) may be either obtained by apheresis procedure from one donor (AP-PC) or may be prepared by pooling the buffy coats (BC-PC) or the platelet-rich plasma of 4-6 whole blood donations [1]. In Switzerland, only AP-PC and $\mathrm{BC}-\mathrm{PC}$ are available. Our regional blood transfusion services usually have a mix of both types of PC in order to optimize the use of whole blood donations, to mitigate the risk of shortages, and to ensure adequate availability of HLA-typed AP-PC. Since the introduction of mandatory universal pathogen inactivation of all PC in 2011 [2], AP-PC and BC-PC are considered clinically equivalent. However, some differences exist between both types of $\mathrm{PC}$, such as the extent of residual contaminating red blood cells (RBC), which has been shown to be considerably lower for AP-PC (0.00017-0.009 ml) compared to BC-PC (0.036-0.59 ml) [3-8].

In comparison to RBC transfusion, where up to $80 \%$ of immunocompetent Rhesus $\mathrm{D}(\mathrm{RhD})$-negative individuals may develop serologically detectable anti-D within 2-5 months after exposition to RhD-positive RBCs [9-13], RhD alloimmunization after PC transfusion is rather uncommon. Earlier studies reported the incidence of $\mathrm{D}$ alloimmunization in $\mathrm{D}$-negative recipients of $\mathrm{D}$-positive PC transfusions to be up to $19 \%[3,14-18]$; more recent studies revealed lower frequencies of $0-7 \%$ [19-23].

Anti-D alloantibodies often may cause severe hemolytic disease of D-positive fetuses/newborns. They also preclude for the recipients to receive $\mathrm{D}$-positive $\mathrm{RBC}$ in times of $\mathrm{D}$-negative $\mathrm{RBC}$ shortage. Based on the reported frequencies of $\mathrm{D}$ alloimmunization in some recent large studies, the administration of Rh Immune Globulin (RhIg) is recommended, if D-mismatched PC prepared from whole blood donations are transfused to D-negative recipients, particularly to females of childbearing potential [8].

\section{KARGER}

() 2018 S. Karger GmbH, Freiburg

Fax +497614520714
PD Dr. med. Behrouz Mansouri, MD Department of Hematology, Inselspital Bern University Hospital, University of Bern 3010 Bern, Switzerland behrouz.mansouri@insel.ch 
Table 1. Demographic and clinical characteristics of the 11 patients

\begin{tabular}{|c|c|c|c|c|c|c|}
\hline Patient-\# & Age / sex & Cause of hospitalization & Therapy resulting in reduced immune function & $\begin{array}{l}\text { ABO } \\
\text { group }\end{array}$ & $\begin{array}{l}\text { Rh } \\
\text { phenotype }\end{array}$ & Pregnancy \\
\hline 1 & $63 / \mathrm{m}$ & $\begin{array}{l}\text { angioimmunoblastic T-cell lym- } \\
\text { phoma }\end{array}$ & $\begin{array}{l}\text { CHOEP chemotherapy (cyclophosphamide, } \\
\text { doxorubicin, vincristine, prednisone, etoposide) }\end{array}$ & A & CCD.ee & - \\
\hline 2 & $80 / \mathrm{f}$ & biologic aortic valve replacement & - & A & ccddee & not known \\
\hline 3 & $73 / f$ & acute myeloid leukemia & cytarabine, daunorubicin & B & CCD.ee & yes (3) \\
\hline 4 & $53 / \mathrm{m}$ & metastatic rectum carcinoma & $\begin{array}{l}\text { FOLFOX chemotherapy (oxaliplatin, folinic acid, } \\
\text { 5-fluorouracil) }\end{array}$ & 0 & ccddee & - \\
\hline 5 & $71 / \mathrm{f}$ & $\begin{array}{l}\text { aortic dissection and aortic } \\
\text { nsufficiency }\end{array}$ & - & A & ccddee & not known \\
\hline 6 & $78 / f$ & $\begin{array}{l}\text { thrombocytopenia of unknown } \\
\text { origin }\end{array}$ & - & $\mathrm{AB}$ & ccddee & not known \\
\hline 7 & $64 / \mathrm{m}$ & multiple myeloma & $\begin{array}{l}\text { high-dose chemotherapy (bendamustine }+ \\
\text { melphalane), autologous stem cell transplantation }\end{array}$ & A & CCD.ee & - \\
\hline 9 & $56 / \mathrm{m}$ & carcinoma testis & $\begin{array}{l}\text { high-dose chemotherapy with carboplatin and } \\
\text { etoposide }\end{array}$ & A & CcD.ee & - \\
\hline 10 & $76 / \mathrm{m}$ & $\begin{array}{l}\text { AML, upper gastrointestinal } \\
\text { bleeding }\end{array}$ & decitabine & A & ccddee & - \\
\hline 11 & $65 / \mathrm{m}$ & acute myeloid leukemia & cytarabine, daunorubicin & 0 & CCD.ee & - \\
\hline
\end{tabular}

There is some evidence that the minimum quantity of RBCs necessary for primary immunization is as low as $0.03-0.05 \mathrm{ml}[8$, $24,25]$. Therefore, a presumed advantage of AP-PC is their lower $\mathrm{RBC}$ content, which is being considered as insufficient for triggering an alloimmunization. On this basis, some centers do not routinely provide RhIg prophylaxis after administration of RhD-positive AP-PC to RhD-negative patients [8].

On the other hand, Kitazawa et al. [24] hypothesized whether also RBC-derived microparticles could trigger alloimmunizations. They estimated that AP-PC contain 0.0001-0.001 ml of RBC-derived microparticles.

$\mathrm{Rh}$ antigens $\mathrm{c}, \mathrm{C}$, e and $\mathrm{E}$ are less immunogenic than $\mathrm{D}$ [13]. So far, there is limited data on PC-induced alloimmunization against other Rh antigens than $\mathrm{D}[7,19]$ and incompatibilities of non-D $\mathrm{Rh}$ antigens have generally been disregarded in BC-PC or AP-PC transfusion [8].

In the context of preparing the hemovigilance report to Swissmedic in 2015, the work-up of a newly formed alloantibody revealed an anti-f specificity, which must have been triggered by RBC antigen exposure through an AP-PC transfusion. This prompted us to implement a systematic prospective assessment of every single newly identified $\mathrm{Rh}$ alloantibody for the putative triggering blood product transfused. With this hemovigilance-induced quality assurance measure, we aimed to identify possible further cases in order to delineate the frequency, the antibody specificities, and the circumstances that may be associated with this phenomenon. We here describe $13 \mathrm{Rh}$ antibodies detected between August 2015 and September 2017 in 11 patients, where Rh alloimmunization has obviously been provoked by platelet transfusion. Furthermore, we compared the frequencies of anti-Rh immunization following $\mathrm{AP}-\mathrm{PC}$ and BC-PC.

\section{Patients and Methods}

As one of the largest tertiary and university hospitals of Switzerland (Inselspital, University Hospital, Insel Group, Bern, Switzerland), we have transfused 18,033 RBC, 7,020 plasma, and 4,371 platelet units in 2016. RBC and platelet products have been provided by the Interregional Blood Transfusion Service Bern, Swiss Red Cross (Bern, Switzerland) (IRB) and the plasma units $\left(\right.$ OctaplasLG ${ }^{\circledR}$ ) by Octapharma (Lachen, Switzerland). In agreement with our national recommendations, we consider BC-PC and AP-PC clinically equivalent, except for HLA- and/or HPA-alloimmunized patients, who mandatorily receive 'best procurable' compatible single donor AP-PC. According to internal standards, a valid serological antibody screen (i.e., performed within the last 96 h) has been mandatory for all patients before any RBC and PC transfusion. For antibody screening and additional antibody identification (indirect antiglobulin and enzyme test), we used BioRad column agglutination technology (BioRad, Cressier, Switzerland). We performed all tests in compliance with the specific requirements of our national guidelines (https://sbsc-bsd.ch/dokuman2/de-de/ bsd/vorschriften/empfehlungen.aspx).

Between August 2015 and September 2017, we found 5,799 positive antibody screening tests in 89,190 immunohematologically analyzed patients. We prospectively registered all newly detected Rh alloantibodies (D and non-D), which could not be explained by incompatible RBC transfusions. For every patient with a newly detected Rh alloantibody we compiled the complete transfusion history. According to antibody specificity, we identified all incompatible PC and/or RBC transfusions. Only patients with newly detected Rh alloantibodies who had not received any cognate $\mathrm{Rh}$ antigen-incompatible RBC transfusion but had been exposed to at least one incompatible PC were included in our study.

Data analyzed of the patients identified to have obviously been alloimmunized through PC transfusions included: age, gender, $\mathrm{ABO}$ blood group and $\mathrm{Rh}$ phenotype, main diagnoses, immunosuppressive drugs, transfusion history (Inselspital only), and date of alloantibody detection [7]. Transfusion history included every RBC and PC unit transfusion.

Recorded data of donors/PC/transfusions included: gender, age, $\mathrm{ABO}$ blood group and Rh phenotype, AP-PC or BC-PC, and date of transfusion. Molecular screening for confirmation of serologically Rh-negative donors is mandatory in Switzerland since 2015 [26].

We performed routine quality control for residual RBC count for $10 \mathrm{AP}-\mathrm{PC}$ and $10 \mathrm{BP}-\mathrm{PC}$ every month using the manual/visual cell counting chamber 
method (Neubauer Chamber). We calculated geometric mean and range including all quality control RBC counts for AP-PC and BC-PC from January 2016 to December 2016.

Based on previous publications, we considered newly detected antibodies following a Rh-incompatible PC transfusion until day 27 as secondary immune response (booster effect) and those found on day 28 or later as a primary immune response against cognate $\mathrm{Rh}$ antigens $[7,8]$.

\section{Results}

\section{Detection of Newly Acquired Rh Antibodies after Platelet}

\section{Transfusion}

From August 2015 to September 2017 a total of 4,596 AP-PC and 5,050 BC-PC were transfused to patients at our institution. In 11 patients (table 1 ) we identified 13 newly acquired $\mathrm{Rh}$ antibodies, which were triggered by a PC transfusion (table 2). Of the newly detected antibodies, 6 were of the specificity anti-D (patients \#2, $\# 4, \# 5$, \#6, \#8, \#10). For patients \#2 and \#6, a booster effect has to be assumed, considering the interval between the last $\mathrm{Rh}$ phenotype-incompatible transfusion and antibody detection. For patient \#5, we interpreted rather a primary immunization because of weak reactivity in the antibody screen and identification tests. Anti-E alloantibodies were detected in 4 cases (patients \#3, \#7, \#9, \#11 (booster effect)), anti-c in 2 cases (patients \#3,\#7), and anti-f in 1 case (patient \#1 (most likely also a booster effect)) (details in table 2). These 11 patients were investigated in detail.

Demographic Data, Underlying Diseases, and Therapy Resulting in Reduced Immune Function

There were 6 males and 5 females with a median age of 67 years (range 53-80 years). Causes of hospitalization were hematological malignancies in 6 cases: Four patients had acute myeloid leukemia, one of them acute promyelocytic leukemia. One patient each had $\mathrm{T}$-angioimmunoblastic leukemia or multiple myeloma. In 1 patient, we documented thrombocytopenia of unclear origin. Solid tumors were documented in 2 patients. Two patients had major heart surgery.

Patients \#1, \#3, \#4, \#7, \#9, and \#11 received cytotoxic chemotherapy; patient $\# 8$ received all-trans-retinoic acid and arsenic trioxide; patient \#10 received demethylating agents. Therefore, 8 out of 11 patients have received treatments that may compromise immune function.

\section{Transfusion History}

The 11 patients received 156 PC (83 BC-PC; 73 AP-PC). Patient $\# 1$ received a single AP-PC, whereas 3 patients (\#2, \#4, and \#6) received BC-PC only. The other 7 patients (\#3, \#5, \#7, \#8, \#9, \#10, and \#11) received both types of $\mathrm{PC}$.

Ten of the 11 patients received $\mathrm{RBC}$ transfusions in addition (total number 152, range 3-41). All RBC units were antigen-negative for the identified alloantibodies and therefore excluded as cause for the alloimmunization.

Because of the known latency of up to 6 months for alloantibody appearance after antigen exposure [27], definite conclusions about the incompatible PC of a series, which may have been the responsible trigger for the individual alloimmunization is not possible. We nonetheless documented the type (BC-PC or AP-PC unit), the number and the time point of the first incompatible transfusion in every patient's transfusion history (details summarized in table 2).

The patients received a total of 75 incompatible $\mathrm{BC}-\mathrm{PC}$ and 31 incompatible AP-PC (table 2). The first incompatible transfusion was in 4 cases an AP-PC and in 9 cases a BC-PC. Five patients (\#2, $\# 4$, \#5, \#6, and \#11) received incompatible BC-PC only, whereof 3 patients received 1 unit each, and 2 patients received 5 units each. Patient \#1 received incompatible AP-PC only (1 unit), whereas 5 patients comprising \#3 and \#7 (with respect to both allo-antibodies), \#8, \#9, and \#10 received incompatible BC-PC as well as APPC. Details are summarized in table 2.

Three of the 11 patients (\#6, \#8, and \#11) already had a known alloantibody and therefore were known to be at a higher risk for additional antibody formation after subsequent transfusions [28-32].

\section{Contamination of $P C$ by $R B C$}

Monitoring the $\mathrm{RBC}$ contamination is a mandatory element of the regular monthly quality controls of PC. There have been no changes of production processes during the study period. The results of the year 2016 showed a mean contamination of 0.304 (range $0.152-1.662$ ) and 0.014 (range $0.003-0.080) \times 10^{9} \mathrm{RBC} / \mathrm{l}$ for $\mathrm{BC}-\mathrm{PC}$ and AP-PC, respectively (table 3 ). Thus, the level of RBC contamination was more than tenfold higher in $\mathrm{BC}-\mathrm{PC}$ than in AP-PC.

\section{Discussion}

As a part of hemovigilance all newly detected alloantibodies have to be reported to the competent authority (Swissmedic). The work-up of such an alloantibody in 2015 revealed an anti-f specificity. Because of the appearance within 3 days, a booster effect seemed reasonable. However, the appearance of the alloantibody surprisingly was induced through an AP-PC transfusion. This prompted us to a prospective assessment of every single newly identified alloantibody with respect to the putative responsible transfused blood unit. Between August 2015 and September 2017, we detected $13 \mathrm{Rh}$ antibodies in 11 patients, which must have been triggered by residual antigen-positive RBC of PC transfusions. Particularly, the spectrum and frequency of non-D Rh antibodies triggered by PC transfusions has exceeded our prospects.

So far, only few publications reported about formation of non-D Rh antibodies after PC transfusion. Kitazawa and colleagues [24] observed 3 patients who developed 4 alloantibodies (2 anti-E, 1 anti- $C$, and 1 anti-c) after platelet transfusions. These patients only received AP-PC. Because RBC contamination in AP-PC (range 0.00017-0.009 $\mathrm{ml} \mathrm{RBC}$ per $\mathrm{PC}$ ) [8], is probably insufficient to trigger alloimmunization (according to literature the minimum is around $0.03-0.05 \mathrm{ml} \mathrm{RBC})[8,24,25,33]$, the authors hypothe- 


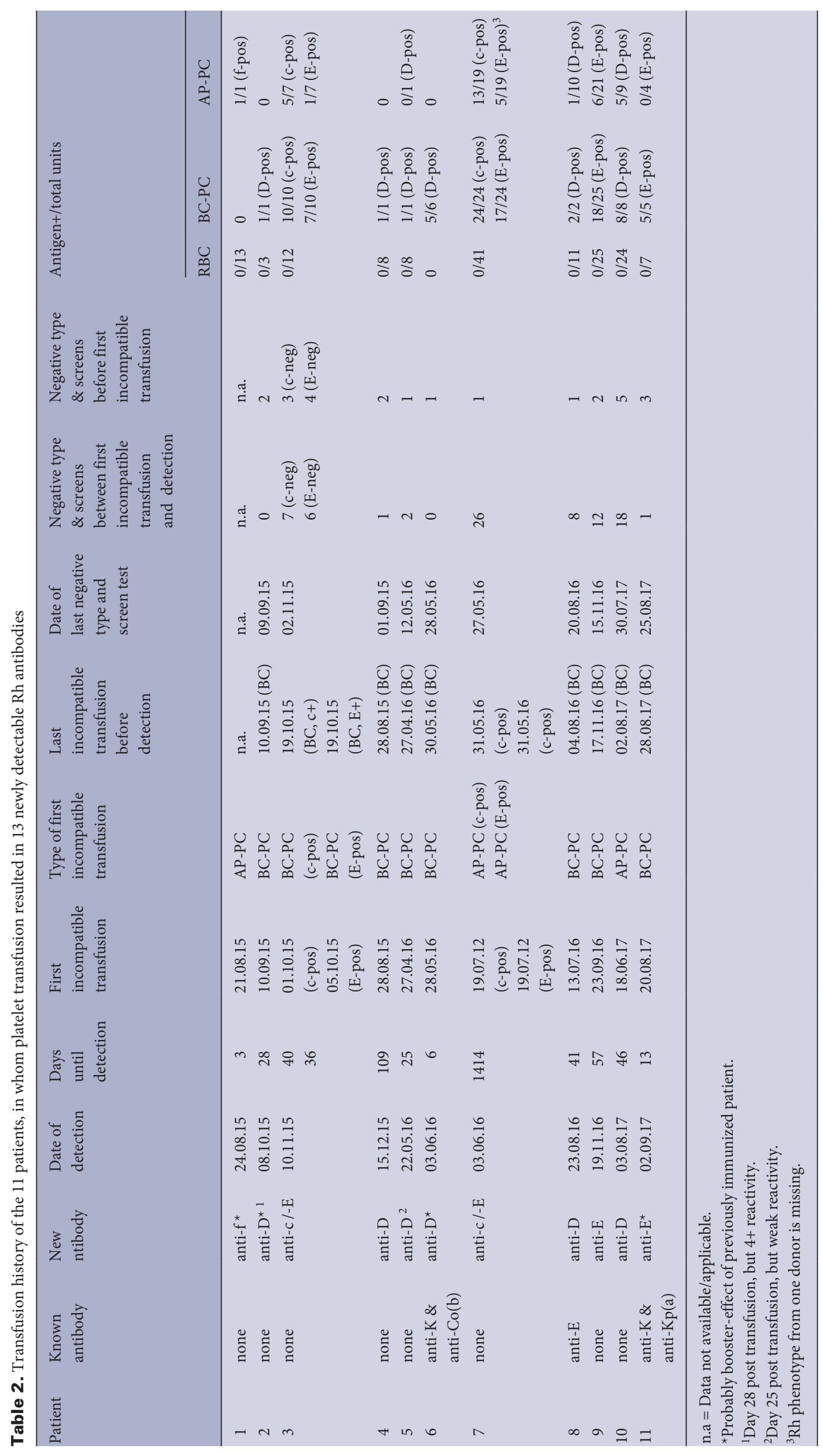


Table 3. Contamination of the PC by RBC (quality controls of 2016)

\begin{tabular}{lll}
\hline PC & Mean (range) RBC $\times 10^{9} / 1$ & $\begin{array}{l}\text { Equates to an estimated } \\
\text { mean blood volume, } \mathrm{ml}\end{array}$ \\
\hline AP-PC & $0.014(0.003-0.080)$ & 0.0028 \\
BC-PC & $0.304(0.152-1.662)$ & 0.0608 \\
\hline
\end{tabular}

sized that RBC microparticles (being present in BC-AP as well as AP-PC) could contribute to alloimmunization. Microparticles can be phagocytosed easily and so are probably more immunogenic than RBCs [34, 35].

In our study we suggest that AP-PC triggers a booster effect of an anti-f alloantibody (\#1), and we therefore suppose that the minimum amount of RBC (and probably also of microparticles) as a trigger of alloimmunization should be critically discussed [24].

According to our quality controls, $\mathrm{RBC}$ contamination was higher in $\mathrm{BC}-\mathrm{PC}$ than in $\mathrm{AP}-\mathrm{PC}$, which was in line with the literature and supports that an increasing antigen dose enhances the potential for immunization [33]. For appraisal of the impact of transfusing either AP-PC or BC-PC (representing different amounts of $\mathrm{RBC}$ contamination) on the risk of Rh alloimmunization, we analyzed in more detail the Rh-incompatible PC transfused. Altogether, the 11 patients described in our investigation received 156 PC (83 BC-PC and 73 AP-PC). The Rh-alloimmunized patients received more incompatible $\mathrm{BC}-\mathrm{PC}(75 / 83)$ than AP-PC (31/73). In the majority of cases, the first incompatible transfusion was a BC-PC (9 out of 13). Finally, 5/11 patients had received incompatible BC-PC only. Due to our limited set of data and the heterogeneity of our small study population, it is not possible to make a reliable assessment about the different alloimmunization risk of either group (BC-PC vs. AP-PC). However, the risk for alloimmunization after BC-PC seems to be higher than after AP-PCs, as supported by previous reports of others [7,33].

More recently, Schonewille and colleagues [27] reported on the incidence of alloantibody formation after $\mathrm{ABO} / \mathrm{RhD}$ or extended matched RBC transfusions. In their randomized trial in surgical patients (MATCH study), they tried to avoid clinically relevant RBC antibody formation by extended (c, C, E, K, Fya, Jka, and S antigens) matched $\mathrm{RBC}$ transfusions. Among the group of patients who received PC (reported to be BC-PC only) in addition to RBCs transfusions, they surprisingly found 6 patients who have formed 8 RBC alloantibodies ( $5 \times$ anti-E, $1 \times$ each anti-D, anti- $K$, and anti-e) after cognate RBC antigen exposure through platelet transfusions only. Moreover, a significant absolute risk difference in favor of extended RBC matching was only shown in the group of patients who did not receive PC transfusions. The authors concluded that $\mathrm{RBC}$-extended matching is only successful if the patient does not receive accompanying non-RBC antigen-matched platelet products [27]. The results of our study with regard to spectrum and frequency of non-D Rh antibodies triggered by platelet transfusion are in line with these reports and strongly support the significant potential of PC transfusions for inducing RBC alloantibodies.

The rate of $\mathrm{RhD}$ alloimmunization is known to depend not only on the RBC content of the PC but also on a variety of further factors, including the immune status of the patient and the rate of secondary immune response [33]. Age (median, 67 years) and gender distribution $(\mathrm{f} / \mathrm{m}=5 / 6)$ of our patients was within expected ranges in the hospital. A high proportion of the patients had either hematological malignancies or solid tumors. They received cytotoxic chemotherapy or other anti-leukemic treatments such as ATRA/ATO or demethylating compounds. The remaining patients had either idiopathic thrombocytopenia or heart surgery (further details in table 1). Our results are in line with the reports of others that $\mathrm{Rh}$ alloimmunization through PC is observed in immunocompetent as well as immunocompromised patients $[7,33]$. The newly detected alloantibodies in our series were most frequently anti-D (6/13), followed by anti-E (4/13), anti-c (2/13), and anti-f (1/13), details in table 2 . In 4 patients ( 2 with anti-D, 1 with anti-E, 1 with anti-f), a booster effect has to be assumed. In patient \#7, there was a gap of 4 years between the last negative type and screen testing and the detection of the alloantibody at our institute. Therefore, an alternative immunization event might have taken place during that period of time.

Our observation underlines the potential of residual RBC in PC for primary immunization or boosting effect against RBC antigens. A secondary immune response is known to arise as a 'boosting' effect after much smaller antigenic provocation than that of the primary response $[27,33]$. In our study population, we can describe a primary or secondary immune response to PC transfusions in 10 out of 11 patients (for \#7 an alternative immunization event cannot be ruled out). In 8 of these 10 patients, a primary immunization can be assumed and in 2/10 patients (\#1 and \#6) a secondary immunization seems to be more likely. Regarding the latter 2 patients, reasons for primary immunization are likely to be from previous transfusions (\#1 and \#6) and/or pregnancies (\#6), but no specific data are available to prove the reason for primary immunization.

In conclusion, BC-PC and AP-PC transfusions may not only induce $\mathrm{RhD}$ alloimmunization, but also immunization against further Rh antigens such as c, E, and f. According to the current literature, the risk seems higher for BC-PC than for AP-PC. Interestingly, in 1 case we found a booster effect of an anti-f alloantibody triggered by one single AP-PC. Therefore, the minimum amount of $\mathrm{RBC}$ as a trigger of alloimmunization (booster effect or primary antibody formation) may be reassessed. Being aware of problems with respect to feasibility, these results may have impact on the next edition of our national guidelines. The responsible national working party will discuss a modification of their recommendations according to $\mathrm{PC}$ transfusion with respect to $\mathrm{Rh}$ and Kell compatibility (e.g. recommendation of AP-PC with extended Rh phenotype and Kell matching for selected patient populations such as females of childbearing age or chronically transfused hemoglobinopathy patients). In addition, remarks about immunologic safety of upper limits of RBC contamination in PC as well as clinical equivalence of BC-AP and AP-PC with respect to immunogenicity will be reconsidered. Our limited set of data does not allow the calculation of a relative risk for alloimmunization through PC. In order to answer this question, larger prospective studies are needed. 


\section{Disclosure Statement}

The authors declare that they have no conflicts of interest relevant to the manuscript submitted.

\section{References}

1 Simon TL, McCullough J, Snyder EL, Solheim BG, Strauss RG (eds): Rossi's Principles of Transfusion Medicine, 5th ed. Chichester, Wiley-Blackwell, 2016.

2 Kaiser-Guignard J, Canellini G, Lion N, Abonnenc M, Osselaer JC, Tissot JD: The clinical and biological impact of new pathogen inactivation technologies on platelet concentrates. Blood Rev 2014;28:235-241.

3 Goldfinger D, McGinniss MH: Rh-incompatible platelet transfusions - risks and consequences of sensitizing immunosuppressed patients. N Engl J Med 1971;284: 942944.

4 SeheultJN, Triulzi DJ, Yazer MH: I am the 9\%: making the case for whole-blood platelets. Transfus Med 2016 26:177-185.

5 Cid J, Lozano M: Risk of Rh(D) alloimmunization after transfusion of platelets from $\mathrm{D}+$ donors to $\mathrm{D}$ - recipients. Transfusion 2005;5:453; author reply 453-454.

6 Cid J, Carbassé G, Pereira A, Sanz C, Mazzara R, Escolar G, Lozano M: Platelet transfusions from D+ donors to D-patients: a 10-year follow-up study of $1014 \mathrm{pa}$ tients. Transfusion 2011;51:1163-1169.

7 Cid J, Lozano M, Ziman A, West KA, O'Brien KL, Murphy MF, Wendel S, Vázquez A, Ortín X, Hervig TA, Delaney M, Flegel WA, Yazer MH; Biomedical Excellence for Safer Transfusion collaborative: Low frequency of anti-D alloimmunization following D+ platelet transfusion: the Anti-D Alloimmunization after D-incompatible Platelet Transfusions (ADAPT) study. Br J Haematol 2015;168:598-603.

8 Cid J, Yaze MH, Lozano M: Platelet transfusion and respecting patient D type. Curr Opin Hematol 2015;22: 540-546.

9 Pollack W, Ascari WQ, Crispen JF, O'Connor RR, Ho TY: Studies on Rh prophylaxis. II. Rh immune prophylaxis after transfusion with Rh-positive blood. Transfusion 1971;11:340-344.

10 Urbaniak SJ, Robertson AE: A successful program of immunizing Rh-negative male volunteers for anti-D production using frozen/thawed blood. Transfusion 1981;21:64-69.

11 Anderson KC, Ness PM: Scientific Basis of Transfusion Medicine: Implications for Clinical Practice. Philadelphia, W.B. Saunders, 2000.

12 Lozano M, Cid J: The clinical implications of platelet transfusions associated with $\mathrm{ABO}$ or $\mathrm{Rh}(\mathrm{D})$ incompatibility. Transfus Med Rev 2003;17:57-68.

13 Urbaniak SJ: Alloimmunity to RhD in humans. Transfus Clin Biol 2006;13:19-22.
4 Lichtiger B, Surgeon J, Rhorer S: Rh-incompatible platelet transfusion therapy in cancer patients. A study of 30 cases. Vox Sang 1983;45:139-143.

15 Baldwin ML, Ness PM, Scott D, Braine H, Kickler TS: Alloimmunization to D antigen and HLA in D-negative immunosuppressed oncology patients. Transfusion 1988;28:330-333.

16 McLeod BC, Piehl MR, Sassetti RJ: Alloimmunization to $\mathrm{RhD}$ by platelet transfusions in autologous bone marrow transplant recipients. Vox Sang 1990;59:185189.

17 Heim MU, Böck M, Kolb HJ, Schleuning M, Mempel $\mathrm{W}$ : Intravenous anti-D gammaglobulin for the prevention of rhesus isoimmunization caused by platelet transfusions in patients with malignant diseases. Vox Sang 1992;62:165-168.

18 Zeiler T, Wittmann G, Zingsem J, Weisbach V, Zimmermann R, Eckstein R: A dose of 100 IU intravenous anti-D gammaglobulin is effective for the prevention of $\mathrm{RhD}$ immunisation after $\mathrm{RhD}$-incompatible single donor platelet transfusion. Vox Sang 1994;66:243.

19 Moncharmont P, Barday G, Meyer F: Red blood cell alloimmunisation after platelet transfusion: a 5-year study. Blood Transfus 2014;12(suppl 1):s147-148.

20 O’Brien KL, Haspel RL, Uhl L: Anti-D alloimmunization after D-incompatible platelet transfusions: a 14year single-institution retrospective review. Transfusion 2014;54:650-654

21 Dunbar NM, Katus MC, Freeman CM, Szczepiorkowski ZM: Easier said than done: $\mathrm{ABO}$ compatibility and $\mathrm{D}$ matching in apheresis platelet transfusions. Transfusion 2015;55:1882-1888.

22 Weinstein R, Simard A4, Ferschke J, Vauthrin M, Bailey JA, Greene M: Prospective surveillance of D- recipients of $\mathrm{D}+$ apheresis platelets: alloimmunization against $\mathrm{D}$ is not detected. Transfusion 2015;55:1327-1330.

23 Cid J, Lozano M, Klein HG, Flegel WA: Matching for the $\mathrm{D}$ antigen in haematopoietic progenitor cell transplantation: definition and clinical outcomes. Blood Transfus 2014;12:301-306.

24 Kitazawa J, Nollet K, Morioka H, Tanaka K, Inomata M, Kubuki Y, Ohto H: Non-D Rh antibodies appearing after apheresis platelet transfusion: stimulation by red cells or microparticles? Vox Sang 2011;100:395-400.

25 Klein HG,Anstee DJ (eds): Mollison's Blood Transfusion in Clinical Medicine. Chichester, Wiley \& Sons, 2005.
Crottet SL, Henny C, Meyer S, Still F, Stolz M, Gottschalk J, Neuenschwander K, Taleghani BM, Gowland P, Frey BM, Fontana S, Hustinx H, Niederhauser C, Gassner C: Implementation of a mandatory donor RHD screening in Switzerland. Transfus Apher Sci 2014;50:169-174.

27 Schonewille H, Honohan Á, van der Watering LM, Hudig F, Te Boekhorst PA, Koopman-van Gemert AW, Brand A: Incidence of alloantibody formation after ABO-D or extended matched red blood cell transfusions: a randomized trial (MATCH study). Transfusion 2016;56:311-320.

28 Tormey CA, Fisk J, Stack G: Red blood cell alloantibody frequency, specificity, and properties in a population of male military veterans. Transfusion 2008 ; 48 : 2069-2076.

29 van de Watering L, Hermans J, Witvliet M, Versteegh M, Brand A: HLA and RBC immunization after filtered and buffy coat-depleted blood transfusion in cardiac surgery: a randomized controlled trial. Transfusion 2003;43:765-771.

30 Schonewille $\mathrm{H}$, van de Watering LM, Brand A: Additional red blood cell alloantibodies after blood transfusions in a nonhematologic alloimmunized patient cohort: is it time to take precautionary measures? Transfusion 2006;46:630-635.

31 Schonewille H, Klumper FJ, van de Watering LM, Kanhai HH, Brand A: High additional maternal red cell alloimmunization after Rhesus- and $\mathrm{K}$-matched intrauterine intravascular transfusions for hemolytic disease of the fetus. Am J Obstet Gynecol 2007;196: 143.e1-6.

32 Schonewille H, de Vries RR, Brand A: Alloimmune response after additional red blood cell antigen challenge in immunized hematooncology patients. Transfusion 2009;49:453-457.

33 Hendrickson JE, Eisenbarth SC, Tormey CA: Red blood cell alloimmunization: new findings at the bench and new recommendations for the bedside. Curr Opin Hematol 2016;23:543-549.

34 Kumpel B: Are weak D RBCs really immunogenic? Transfusion 2006;46:1061-1062; discussion 10621066

35 Canellini G, Rubin O, Delobel J, Crettaz D, Lion N, Tissot JD: Red blood cell microparticles and blood group antigens: an analysis by flow cytometry. Blood Transfus 2012;10(suppl 2):s39-45. 